\title{
Modelling Atwood's Machine with Three Degrees of Freedom
}

\author{
Alexander N. Prokopenya
}

Received: 1 December 2017 / Revised: 20 April 2018 / Accepted: 21 May 2018 / Published online: 6 July 2018

(C) The Author(s) 2018

\begin{abstract}
A generalized model of the Atwood machine when two bodies can swing in a plane is considered. Combining symbolic and numerical calculations, we have obtained equations of motion of the system and analyzed their solutions. We have shown that oscillations can completely modify motion of the system while the simple Atwood machine demonstrates only the uniformly accelerated motion of the bodies. In particular, a quasi-periodic motion of the system can take place even in case of equal masses of the bodies. We have also obtained a differential equation determining an averaged translational motion of the system and have shown that its solution corresponds completely to the numerical solution of the exact differential equations of motion. The validity of the results obtained is demonstrated by means of the simulation of motion of swinging Atwood's machine with the computer algebra system Wolfram Mathematica.
\end{abstract}

Keywords Atwood's machine · Oscillation · Quasi-periodic motion · Averaged motion · Simulation · Wolfram mathematica

Mathematics Subject Classification Primary 34A34; Secondary 34C60

\section{Introduction}

The uniformly accelerated motion is traditionally considered in the course in physics and the Atwood machine was proposed to demonstrate just such kind of motion (see [1]). In the simplest case it consists of two bodies, having masses $m_{1}$ and $m_{2}\left(m_{2}>m_{1}\right)$, attached to opposite ends of a massless inextensible thread wound round a massless frictionless pulley. If each body is constrained to move only along a vertical and the thread doesn't slip on the pulley, the Atwood machine has only one degree of freedom. Using Newton's second law, one can easily obtain the following expression for the acceleration of the bodies:

$a=\frac{m_{2}-m_{1}}{m_{2}+m_{1}} g$

where $g$ is a gravity acceleration.

A. N. Prokopenya $(\varangle)$

Warsaw University of Life Sciences - SGGW, Nowoursynowska str. 159, 02-776 Warsaw, Poland

e-mail: alexander_prokopenya@sggw.pl 
However, in real experiment the bodies, being hung on a flexible thread, can oscillate during their motion under an influence of the force of gravity. In such a case the Atwood machine has more than one degree of freedom and its motion is described by a complex system of nonlinear differential equations. Although a general solution of this system can not be found in symbolic form, one can choose some realistic values of the system parameters and solve the equations numerically. Such simulation and real experiments show that the oscillations can modify the system motion significantly and some unexpected kinds of motion such as a quasi-periodic or chaotic motion can arise (see [2-9]).

To clarify the physical reasons of such influence of oscillation on the system motion in the previous paper [10] we considered the simplest generalization of the Atwood machine when only one body is allowed to swing in a plane while the other body can move only along a vertical. We have shown that oscillation results in increasing of an averaged tension of the thread which depends on the amplitude of oscillation. If the amplitude becomes large enough the averaged tension exceeds the gravity force $m_{2} g$, and the body of the smaller mass $m_{1}$ can pull the body $m_{2}$ up, which is not possible in the system without oscillation. As a result a quasi-periodic motion of the system can occur.

In the present paper we consider the more complicated Atwood machine when both bodies are allowed to swing in the plane. Such a system has three degrees of freedom and can demonstrate different kinds of quasi-periodic motion depending on the mass difference and initial conditions. Note that the equations of motion become more complicated and their analysis requires to combine symbolic and numerical calculations. However, such analysis can be successfully performed with the computer algebra system Mathematica (see [11]) that is used for doing all relevant calculations and visualization of results.

\section{Equations of Motion}

Let us consider a generalization of Atwood's machine when both bodies are allowed to swing in a plane. To eliminate an influence of the pulley mass and size on the system motion and to take into account only oscillations of the bodies we replace the pulley in the standard Atwood machine by two separated small massless pulleys (see Fig. 1). The corresponding mechanical system has three degrees of freedom and its geometrical configuration can be described in terms of three variables, namely, two angles $\varphi_{1}, \varphi_{2}$ determining deviation of the thread from a vertical in the left-hand and right-hand sides of the system, and a length $r$ of the thread between the body $m_{1}$ and the point, where the thread departs from the pulley. Then Cartesian coordinates $x_{1}, y_{1}, x_{2}, y_{2}$ of the bodies can be determined as

$x_{1}(t)=r(t) \sin \varphi_{1}(t), \quad y_{1}(t)=-r(t) \cos \varphi_{1}(t)$,
$x_{2}(t)=-b+(L-r(t)) \sin \varphi_{2}(t), \quad y_{2}(t)=-(L-r(t)) \cos \varphi_{2}(t)$,

where the distance between the pulleys $b$ does not influence the dynamics of the system and is chosen from the condition of avoiding collisions of the bodies. Note that the radius of each pulley is considered to be negligibly small and so the total length of the thread is equal to $L+b$.

Doing standard symbolic calculation, we can write the Lagrangian function of the system as (see, for example, [12])

$$
\begin{aligned}
\mathcal{L}= & \frac{1}{2} \dot{r}^{2}+\frac{1}{2(1+\mu)}\left(r^{2} \dot{\varphi}_{1}^{2}+(L-r)^{2} \dot{\varphi}_{2}^{2}\right) \\
& +\frac{g}{1+\mu}\left(r \cos \varphi_{1}+\mu(L-r) \cos \varphi_{2}\right),
\end{aligned}
$$

where the dot in $\dot{x}$ denotes the time derivative of the function $x(t)$, and $\mu=m_{2} / m_{1}$ is the system parameter. Note that the corresponding Hamiltonian given by 
Fig. 1 Generalized Atwood's machine with two oscillating bodies

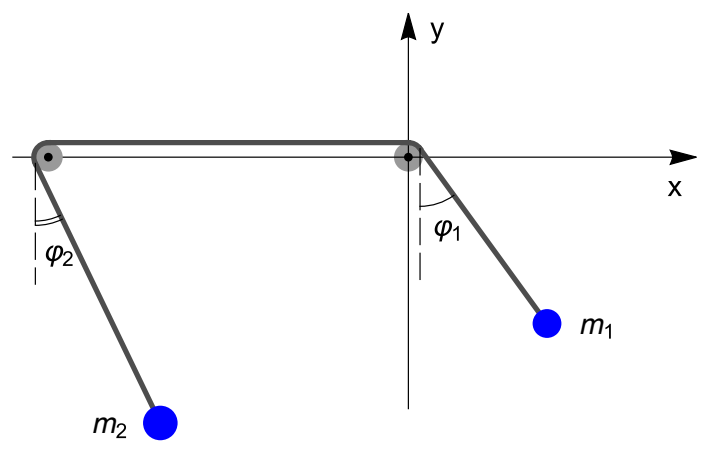

$$
\begin{aligned}
\mathcal{H} & =\frac{p_{r}^{2}}{2}+\frac{1+\mu}{2}\left(\frac{p_{\varphi 1}^{2}}{r^{2}}+\frac{p_{\varphi 2}^{2}}{(L-r)^{2}}\right) \\
& -\frac{g}{1+\mu}\left(r \cos \varphi_{1}+\mu(L-r) \cos \varphi_{2}\right)=\text { const },
\end{aligned}
$$

is an integral of motion. Here $p_{r}, p_{\varphi 1}$, and $p_{\varphi 2}$ are the momenta canonically conjugated to coordinates $r, \varphi_{1}$, and $\varphi_{2}$, respectively.

Using the Lagrangian (2.1) or the Hamiltonian (2.2), we obtain the equations of motion of the system in the form

$$
\begin{aligned}
(1+\mu) \ddot{r} & =g\left(\cos \varphi_{1}-\mu \cos \varphi_{2}\right)+r \dot{\varphi}_{1}^{2}-\mu(L-r) \dot{\varphi}_{2}^{2}, \\
r \ddot{\varphi}_{1} & =-g \sin \varphi_{1}-2 \dot{r} \dot{\varphi}_{1}, \\
(L-r) \ddot{\varphi}_{2} & =-g \sin \varphi_{2}+2 \dot{r} \dot{\varphi}_{2} .
\end{aligned}
$$

Obviously, in the absence of oscillations when $\varphi_{1}=\varphi_{2} \equiv 0$, Eqs. (2.4), (2.5) are satisfied identically while Eq. (2.3) is easily solved and its solution is given by

$r(t)=r_{0}+v_{0} t-\frac{g(\mu-1) t^{2}}{2(\mu+1)}$,

where $r_{0}=r(0)$ and $v_{0}=\dot{r}(0)$ are initial distance and velocity, respectively. In case of $\mu \neq 1$ solution (2.6) determines a uniformly accelerated motion of the bodies.

\section{Motion of Atwood's Machine with Two Oscillating Bodies}

Oscillation of even only one body complicates the equations of motion significantly and may result in appearance of a quasi-periodic motion of the system. In case of the two oscillating bodies the equations of motion (2.3)-(2.5) are much more complicated in comparison to the case of one body oscillation (see [10]). And to demonstrate some new peculiarities of the system motion we have to look for numerical solutions of these equations for different initial conditions and different values of the system parameter $\mu$.

\subsection{Equal Masses}

First let us consider the case when both bodies have the same mass $(\mu=1)$ and the system is in the state of equilibrium at the initial instant of time. The corresponding initial conditions are given by

$$
r(0)=r_{0}, \quad \dot{r}(0)=0, \quad \varphi_{1}(0)=\varphi_{10}, \quad \varphi_{2}(0)=\varphi_{20}, \quad \dot{\varphi}_{1}(0)=0, \quad \dot{\varphi}_{2}(0)=0 .
$$



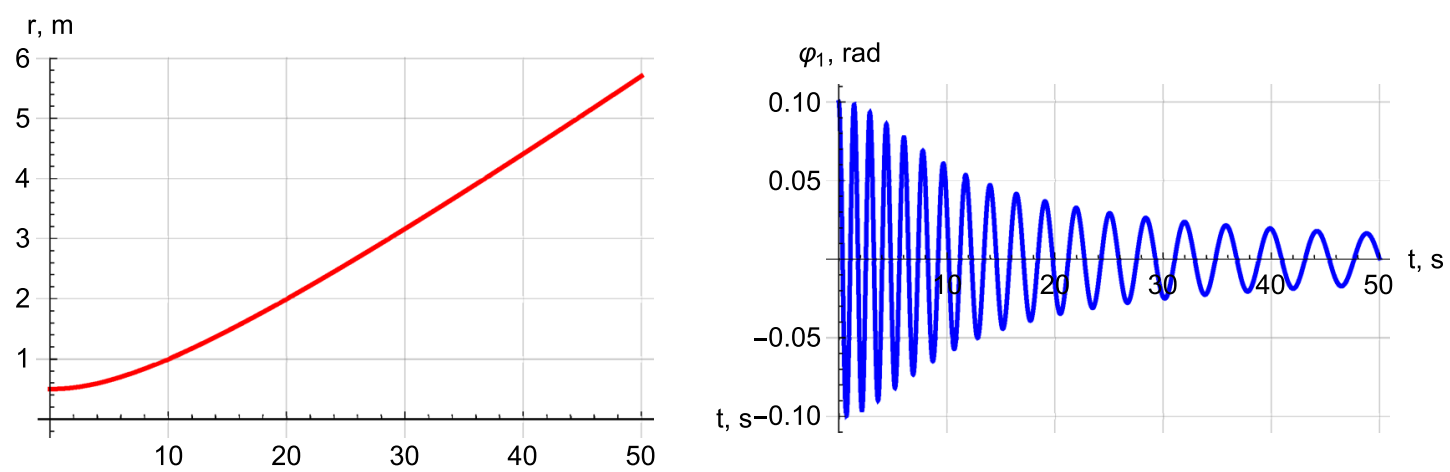

Fig. 2 Motion of the system in case of equal masses and one oscillating body: $L=1.5, r_{0}=0.5, \varphi_{10}=0.1, \varphi_{20}=0$

Fig. 3 Translational motion of the system in case of two oscillating bodies: $\mu=1$, $r_{0}=0.9, \varphi_{10}=0.08$, $\varphi_{20}=0.05$

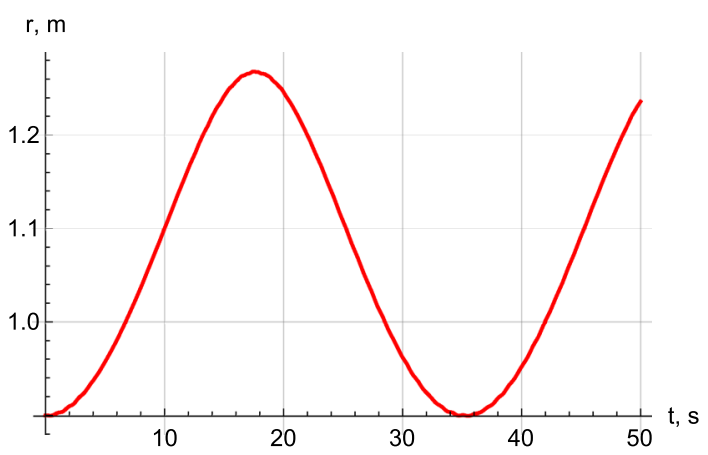

In case of $\varphi_{10}>0$ and $\varphi_{20}=0$ only the first body can oscillate. Using the Mathematica built-in function NDSolve (see [11]), we obtain the corresponding numerical solution of the equations of motion (2.3)-(2.5) (see Fig. 2). It shows that $r(t)$ is an increasing nonlinear function and, therefore, the oscillating body $m_{1}$ pulls the second body $m_{2}$ up. Note that it is not possible in case of the simple Atwood machine because for equal masses $m_{1}=m_{2}$ the acceleration (1.1) is equal to zero and for the initial conditions (3.1) we obtain $r(t)=r_{0}=$ const (see (2.6)). Only in case of $\dot{r}(0)=v_{0}>0$ the simple Atwood machine can demonstrate a uniform motion, but the function $r(t)$ shown in Fig. 2 is not linear.

If both bodies are deviated from the equilibrium $\left(\varphi_{10}>\varphi_{20}>0\right)$ the system motion completely changes (see Figs. 3, 4). One can readily see that at the beginning an amplitude of oscillation of the body $m_{1}$ is greater than that of the body $m_{2}$ and so it pulls the body $m_{2}$ up. However, the length of the thread between the body $m_{1}$ and the pulley increases while the length of the thread between the body $m_{2}$ and the second pulley decreases. This results in decreasing the amplitude of oscillation of the body $m_{1}$ while the amplitude of the body $m_{2}$ oscillation increases. Consequently, the growth of the function $r(t)$ slows down and after reaching a maximum it starts to decrease. Then the bodies change their roles and the second body $m_{2}$, oscillating with greater amplitude, starts to move down and to pull body $m_{1}$ up and so on. Thus, the system demonstrates quasi-periodic motion which is quite difficult to predict without solving the equations of motion.

Choosing symmetrical initial conditions $r_{0}=L / 2, \varphi_{10}=-\varphi_{20}, r_{0}=L-r_{0}=L / 2$ and solving the equations of motion, we obtain the results shown in Fig. 5. One can readily see that the distance $r(t)$ does not change with time while the bodies oscillate in opposite phases with equal amplitudes. Intuitively this could be expected because of the symmetry of the system. It should be emphasized also that the system does not demonstrate translational motion and increasing the accuracy of calculation does not change the graphs shown in Fig. 5. 

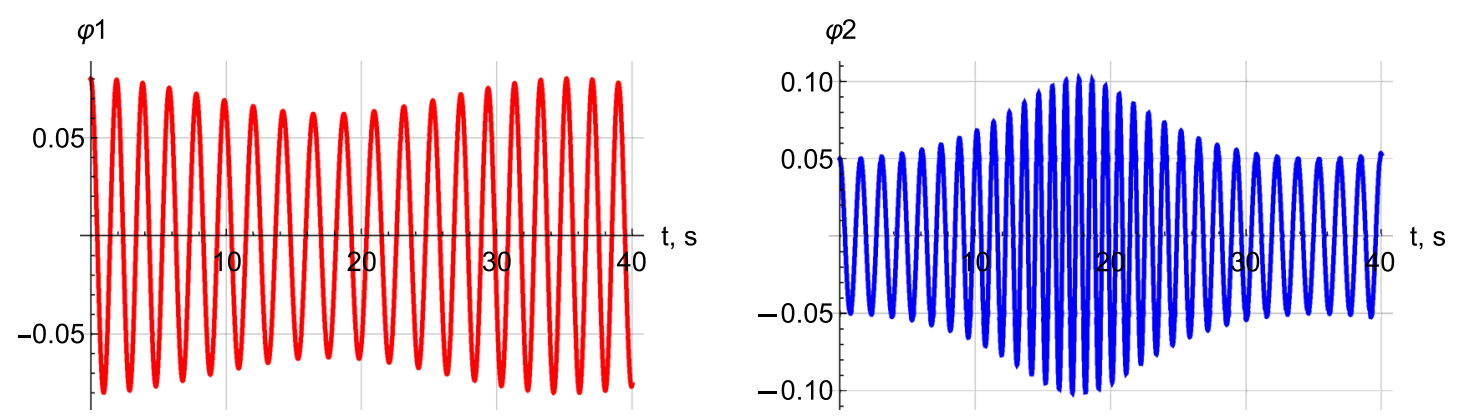

Fig. 4 Oscillations of the bodies: $\mu=1$
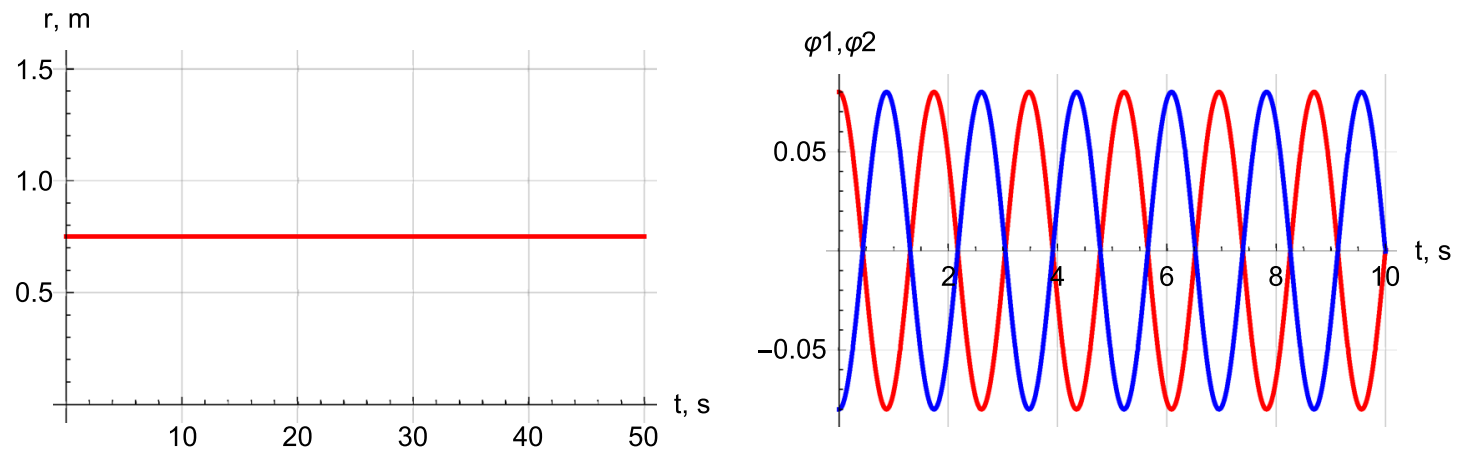

Fig. 5 Motion of the system in case of $\mu=1, r_{0}=L / 2=0.75, \varphi_{10}=-\varphi_{20}=0.08$
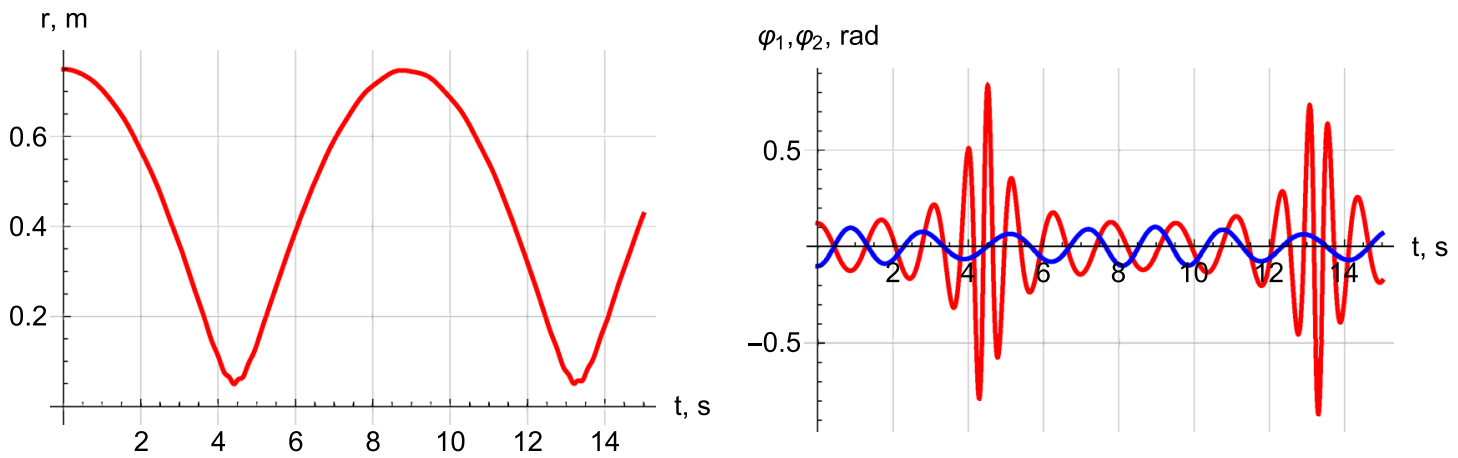

Fig. 6 Motion of the system in case of $\mu=1.02, r_{0}=L / 2, \varphi_{10}=0.12, \varphi_{20}=-0.1\left(\varphi_{1}(t)-\right.$ red, $\varphi_{2}(t)-$ blue $)($ color figure online)

\subsection{Unequal Masses $(\mu \neq 1)$}

Increasing the mass of one body ( $m_{2}>m_{1}$, for example) breaks the symmetry of the system taking place in case of the initial conditions $r_{0}=L / 2, \varphi_{10}=-\varphi_{20}$. The heavier body $m_{2}$ starts to move down and to pull the body $m_{1}$ up even if the initial deviation of the body $m_{1}$ is a little bit greater than that of the body $m_{2}$ (see Fig. 6). As the length $L-r(t)$ of the thread between the body $m_{2}$ and the pulley increases the amplitude of its oscillation decreases. At the same time the amplitude of the body $m_{1}$ oscillation grows up because the length of the thread between this body and the pulley decreases. When this amplitude becomes sufficiently large, the bodies stop their translational motion and start to move in opposite direction. Again we observe a quasi-periodic motion of the system.

Increasing the initial deviation of the body $m_{1}$, one can observe that for sufficiently large value of $\varphi_{10}$ the distance $r(t)$ starts to increse and the body $m_{1}$ pulls up the heavier body $m_{2}$ (see Fig. 7). Again, when the amplitude of its oscillation becomes small enough, the translational motion of the bodies slows down and further they start to move 

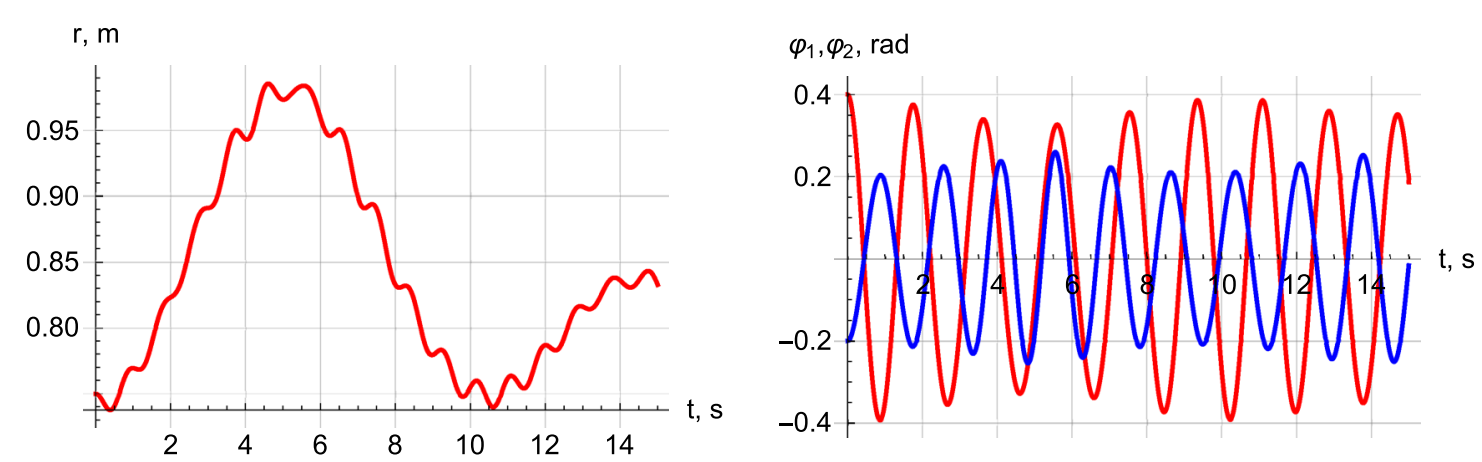

Fig. 7 Motion of the system in case of $\mu=1.02, r_{0}=L / 2, \varphi_{10}=0.4, \varphi_{20}=-0.2$

in the opposite direction. When the body $m_{1}$ reaches its initial position, the amplitude of its oscillation becomes large enough to change the direction of translational motion of the system again and so on. Oscillation of the heavier body $m_{2}$ modifies the system motion a little bit but its character remains the same.

Note that if the difference of masses of the bodies $m_{1}$ and $m_{2}$ is small enough and both bodies acquire nonzero initial angular velocities, the system demonstrates quasi-periodic motion. Only for large enough difference of masses quasi-periodic motion may disappear, because the length of the thread is finite and one of the bodies can reach the pulley before the amplitude of its oscillation becomes sufficiently large to stop translational motion of the system.

\section{Qualitative Analysis of the System Motion}

Numerical solutions of Eqs. (2.3)-(2.5) visualized in Figs. 2, 3, 4 and 5 demonstrate clearly that even small oscillations of the bodies can modify substantially the character of the system motion. Therefore, it makes sense to start investigation of these equations from the simplified case when the oscillations are considered to be small. Assuming $\left|\varphi_{1,2}(t)\right| \ll 1$, one can expand trigonometric functions in (2.3)-(2.5) in power series in terms of $\varphi_{1}, \varphi_{2}$ up to the second order and to rewrite the equations of motion of the system in the form

$$
\begin{aligned}
\ddot{r} & =\frac{g(1-\mu)}{1+\mu}-\frac{g}{2(1+\mu)}\left(\varphi_{1}^{2}-\mu \varphi_{2}^{2}\right)+\frac{1}{1+\mu}\left(r \dot{\varphi}_{1}^{2}-\mu(L-r) \dot{\varphi}_{2}^{2}\right), \\
\ddot{\varphi}_{1} & =-\frac{g}{r} \varphi_{1}-\frac{2 \dot{r}}{r} \dot{\varphi}_{1}, \\
\ddot{\varphi}_{2} & =-\frac{g}{L-r} \varphi_{2}+\frac{2 \dot{r}}{L-r} \dot{\varphi}_{2} .
\end{aligned}
$$

In spite of the simplification, Eqs. (4.1)-(4.3) are quite complicated and application of the Mathematica built-in functions (see [11]) does not enable us to find their general solution. However, one can try to find some particular solutions of Eqs. (4.1)-(4.3) and to investigate behaviour of the system in their neighbourhood.

First, let us check whether a stationary solution $r(t)=r_{0}=$ const may exist. Obviously, in such a case $\dot{r}=0$ and Eqs. (4.2), (4.3) describe harmonic oscillations of the bodies. The corresponding solutions satisfying the initial conditions (3.1) are

$$
\begin{aligned}
& \varphi_{1}(t)=\varphi_{10} \cos \left(\sqrt{\left.\frac{g}{r_{0}} t\right),}\right. \\
& \varphi_{2}(t)=\varphi_{20} \cos \left(\sqrt{\frac{g}{L-r_{0}}} t\right) .
\end{aligned}
$$

On substituting $r(t)=r_{0}=$ const and solutions (4.4), (4.5) into (4.1) and reducing the trigonometric functions, we obtain the following equation 
$1-\mu+\frac{1}{4}\left(\varphi_{10}^{2}-\mu \varphi_{20}^{2}\right)-\frac{3}{4}\left(\varphi_{10}^{2} \cos \left(2 \sqrt{\frac{g}{r_{0}}} t\right)-\mu \varphi_{20}^{2} \cos \left(2 \sqrt{\frac{g}{L-r_{0}}} t\right)\right)=0$.

Obviously, Eq. (4.6) may be satisfied for any instant of time only if the following conditions are fulfilled

$\varphi_{10}^{2}=\mu \varphi_{20}^{2}, \quad r_{0}=L-r_{0}$.

However, the first term $(1-\mu)$ in (4.6) vanishes only if $\mu=1$ or the bodies have the same masses. Therefore, the stationary solution $r(t)=r_{0}=$ const exists only in the case when $m_{1}=m_{2}$ and two pendulums in the left- and the right-hand side of the system shown in Fig. 1 have the same length $r_{0}=L / 2$. Besides, the amplitudes of their oscillations must be the same and the bodies should oscillate in phase or in counter phase. Owing to the smallness of the pulley radius these two kinds of oscillations influence the system motion in the same way.

One can easily check that such stationary solution satisfies also the exact equations of motion (2.3)-(2.5) if $\mu=1$. Indeed, in case of $r(t)=r_{0}=$ const and $\dot{r}=0$ Eqs. (2.4), (2.5) determine nonlinear oscillations of two pendulums which can be described in terms of the elliptic functions. In case of $r_{0}=L / 2$ the pendulums have the same frequency of oscillations and we have $\varphi_{1}(t)= \pm \varphi_{2}(t)$ for any instant of time if the initial conditions $\varphi_{10}= \pm \varphi_{20}$ are chosen (see (3.1)). As a result equation (2.3) is satisfied identically. Note that the numerical solution of the equations of motion (2.3)-(2.5) shown in Fig. 5 demonstrates just this kind of motion.

In case of $\varphi_{10} \neq \pm \varphi_{20}$ or $r_{0} \neq L-r_{0}$ or $\dot{r}_{0} \neq 0$ or $\mu \neq 1$ the symmetry of the system is broken and it demonstrates quasi-periodic motion. However, one can observe that translational motion as well as oscillations of the bodies may be considered as superposition of two kinds of motion one of which is relatively slow while another one is fast (see Figs. 3, 4, 6, 7). For example, the amplitude and frequency of oscillation of each body change slowly in time and their changes for one period of oscillation are quite small. Then we can represent the corresponding solutions of Eqs. (4.2), (4.3) in the form

$\varphi_{1}(t)=A_{1}(t) \cos \left(\sqrt{\frac{g}{r_{1}(t)}} t\right), \varphi_{2}(t)=A_{2}(t) \cos \left(\sqrt{\frac{g}{L-r_{1}(t)}} t\right)$,

where $A_{1}(t), A_{2}(t), r_{1}(t)$ are unknown functions. Here we have taken into account that solution of equation (4.1) may be also represented as $r(t)=r_{1}(t)+r_{2}(t)$, where the function $r_{1}(t)$ describes slow change of the distance $r(t)$, while $r_{2}(t)$ corresponds to its fast change due to oscillations of the bodies, and $\left|r_{2}(t)\right| \ll r_{1}(t)$ (see Figs. 3, 6). Note that the average value of the function $r_{2}(t)$ for the period of its oscillation may be considered as negligibly small, while the change of the function $r_{1}(t)$ is very small for this time. Therefore, the function $r_{1}(t)$ describes a smooth averaged translational motion of the system and its derivative as well as derivatives of the functions $A_{1}(t), A_{2}(t)$ satisfy the condition

$\frac{\dot{r}_{1}(t)}{r_{1}(t)} \ll \sqrt{\frac{g}{r_{1}(t)}}, \frac{\dot{A}_{1}(t)}{A_{1}(t)} \ll \sqrt{\frac{g}{r_{1}(t)}}, \frac{\dot{A}_{2}(t)}{A_{2}(t)} \ll \sqrt{\frac{g}{r_{1}(t)}}$,

where $\sqrt{g / r_{1}(t)}$ determines a frequency of the body $m_{1}$ oscillation, and both frequencies $\sqrt{g / r_{1}(t)}, \sqrt{g /\left(L-r_{1}(t)\right)}$ may be considered as values of the same order.

On substituting the solutions (4.8) into Eqs. (4.2), (4.3) and keeping only the leading terms, we obtain two differential equations

$$
\begin{aligned}
& -\left(2 \dot{A}_{1}(t) r_{1}(t)+\frac{3}{2} A_{1}(t) \dot{r}_{1}(t)\right) \sqrt{\frac{g}{r_{1}(t)}} \sin \left(\sqrt{\frac{g}{r_{1}(t)}}\right)=0, \\
& -\left(2 \dot{A}_{2}(t)\left(L-r_{1}(t)\right)-\frac{3}{2} A_{2}(t) \dot{r}_{1}(t)\right) \sqrt{\frac{g}{L-r_{1}(t)}} \sin \left(\sqrt{\frac{g}{L-r_{1}(t)}}\right)=0,
\end{aligned}
$$


where the conditions (4.9) were taken into account. Integrating the Eqs. (4.10), (4.11) gives

$A_{1}(t)=\varphi_{10}\left(\frac{r_{0}}{r_{1}(t)}\right)^{3 / 4}, A_{2}(t)=\varphi_{20}\left(\frac{L-r_{0}}{L-r_{1}(t)}\right)^{3 / 4}$,

where $r_{1}(t)$ is an unknown function which describes a smooth translational motion of the system.

Note that on substituting $r(t)=r_{1}(t)+r_{2}(t)$ and solutions (4.8) into Eq. (4.1) we obtain

$$
\begin{aligned}
\ddot{r}_{1}(t)+\ddot{r}_{2}(t)= & \frac{g(1-\mu)}{1+\mu} \\
& -\frac{g}{2(1+\mu)}\left(A_{1}^{2} \cos ^{2}\left(\sqrt{\frac{g}{r_{1}(t)}} t\right)-\mu A_{2}^{2} \cos ^{2}\left(\sqrt{\frac{g}{L-r_{1}(t)}} t\right)\right) \\
& +\frac{1}{1+\mu}\left(\left(r_{1}+r_{2}\right)\left(\frac{d}{d t}\left(A_{1} \cos \left(\sqrt{\frac{g}{r_{1}(t)}} t\right)\right)\right)^{2}\right. \\
& \left.-\mu\left(L-r_{1}-r_{2}\right)\left(\frac{d}{d t}\left(A_{2} \cos \left(\sqrt{\frac{g}{L-r_{1}(t)}} t\right)\right)\right)^{2}\right) .
\end{aligned}
$$

Performing symbolic calculations in the right-hand side of Eq. (4.13), we obtain the terms of two different kinds, namely, the terms determining a smooth change of the function $r_{1}(t)$ and the terms having the sine and cosine functions as multipliers which determine fast oscillation of the function $r_{2}(t)$. Separating the slow and the fast terms in the left- and the right-hand side of Eq. (4.13) and keeping only the leading terms, we obtain two equations

$$
\begin{aligned}
\ddot{r}_{1}(t)= & \frac{g}{1+\mu}\left(1-\mu+\frac{\varphi_{10}^{2}}{4}\left(\frac{r_{0}}{r_{1}}\right)^{3 / 2}-\frac{\mu \varphi_{20}^{2}}{4}\left(\frac{L-r_{0}}{L-r_{1}}\right)^{3 / 2}\right), \\
\ddot{r}_{2}(t)= & -\frac{3 g}{4(1+\mu)}\left(\varphi_{10}^{2}\left(\frac{r_{0}}{r_{1}(t)}\right)^{3 / 2} \cos \left(2 \sqrt{\frac{g}{r_{1}(t)}} t\right)\right. \\
& \left.-\mu \varphi_{20}^{2}\left(\frac{L-r_{0}}{L-r_{1}(t)}\right)^{3 / 2} \cos \left(2 \sqrt{\frac{g}{L-r_{1}(t)}} t\right)\right),
\end{aligned}
$$

where conditions (4.9) and Eq. (4.12) were taken into account.

Obviously, Eq. (4.14) is independent of the function $r_{2}(t)$ and may be solved. One integration of this equation gives

$\frac{1}{2} \dot{r}_{1}^{2}-\frac{g}{1+\mu}\left((1-\mu) r_{1}-\frac{\varphi_{10}^{2} r_{0}}{2} \sqrt{\frac{r_{0}}{r_{1}}}-\frac{\mu \varphi_{20}^{2}\left(L-r_{0}\right)}{2} \sqrt{\frac{L-r_{0}}{L-r_{1}}}\right)=C=$ const,

where the constant $C$ is determined from the initial conditions. In case of the bodies of equal masses and initial conditions $\varphi_{10}=\varphi_{20}, \dot{\varphi}_{10}=\dot{\varphi}_{20}=0, r_{0}=L / 2$, Eq. (4.16) takes the form

$\dot{r}_{1}^{2}=\dot{r}_{1}^{2}(0)+\frac{g r_{0} \varphi_{10}^{2}}{2}\left(2-\sqrt{\frac{r_{0}}{r_{1}}}-\sqrt{\frac{r_{0}}{2 r_{0}-r_{1}}}\right)$.

As expression in the parenthesis in the right-hand side of (4.17) is non-positive and is equal to zero only for $r_{1}=r_{0}$, it follows from the condition $\dot{r}_{1}^{2} \geq 0$ that in case of $\dot{r}_{1}(0)=0$ Eq. (4.16) has only a stationary solution $r_{1}(t)=r_{0}$ shown in Fig. 5. For nonzero initial velocity $\dot{r}_{1}(0) \neq 0$ we observe slow oscillation of the function $r_{1}(t)$ near the point $r_{1}=r_{0}$ in the domain determined by the condition $\dot{r}_{1}^{2} \geq 0$. This result corresponds completely to the numerical solution of the equations of motion (4.1)-(4.3) (see Fig. 8). The corresponding functions $\varphi_{1}(t), \varphi_{2}(t)$ 
Fig. 8 Solution of Eq. (4.14) (dashed blue curve) and numerical solution of the equations of motion (4.1)-(4.3) (solid red curve) in case of $\mu=1$, $\varphi_{10}=\varphi_{20}=0.05$, $\dot{r}(0)=0.02$
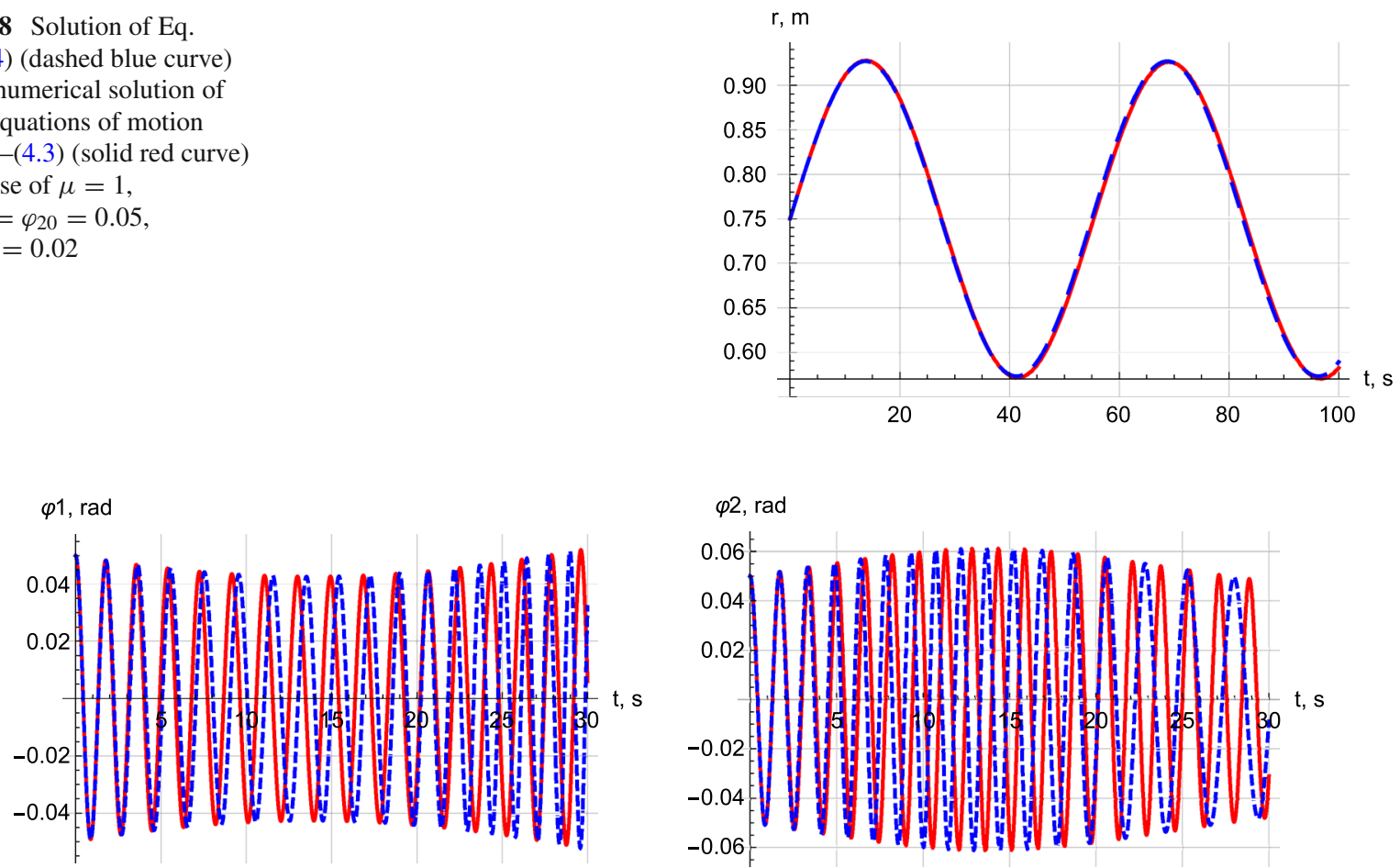

Fig. 9 Oscillations of the bodies described by solutions (4.8) (dashed blue curves) and numerical solutions of the equations of motion (4.1)-(4.3) (solid red curve), $\mu=1, \varphi_{10}=\varphi_{20}=0.05, \dot{r}(0)=0.02$

Fig. 10 Solution of Eq. (4.14) (dashed blue curve) and numerical solution of the equations of motion (4.1)-(4.3) (solid red curves) in case of $\mu=1.02$, $r_{0}=L / 2=0.75$, $\varphi_{10}=-\varphi_{20}=0.1$

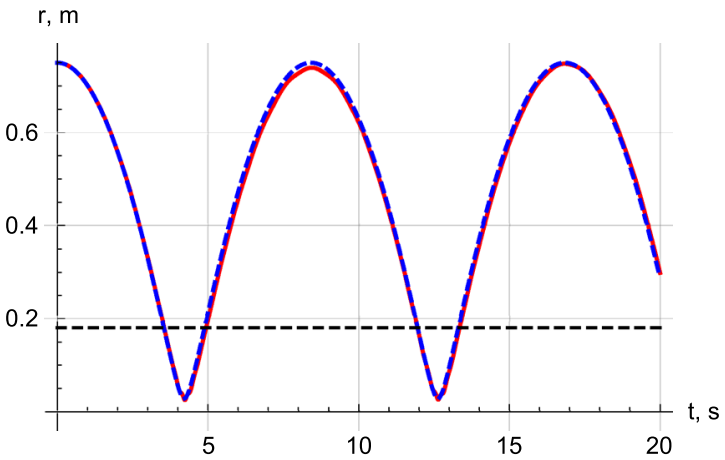

given by (4.12) are depicted in Fig. 9. Of course, expressions (4.12) give only an approximation for the solutions but qualitatively they demonstrate the same behaviour as numerical solutions of the equations of motion (4.1)-(4.3).

Note that the equilibrium value of $r_{1}$ which corresponds to zero of the right-hand side in Eq. (4.14) depends on the parameter $\mu$ and in case of $\mu>1$ it becomes smaller than $r_{0}$. However, the solution of Eq. (4.14) shown in Fig. 10 is in a good agreement with the corresponding solution of the equations of motion (4.1)-(4.3). The equilibrium value of $r_{1}$ is depicted in Fig. 10 by the dashed black line.

As soon as the function $r_{1}(t)$ has been found the function $r_{2}(t)$ describing small oscillation of the distance $r(t)$ is obtained as solution of Eq. (4.15). Using the NDSolve Mathematica function, one can easily find the corresponding numerical solution. However, taking into account a small change of the function $r_{1}(t)$ for a period of oscillation, one can easily integrate Eq. (4.15) under the assumption that $r_{1}(t)$ is a constant. The corresponding approximate solution of Eq. (4.15) is given by 


$$
\begin{aligned}
r_{2}(t)= & \frac{3}{16(1+\mu)}\left(r_{0} \varphi_{10}^{2} \sqrt{\frac{r_{0}}{r_{1}(t)}} \cos \left(2 \sqrt{\frac{g}{r_{1}(t)}} t\right)\right. \\
& \left.-\mu\left(L-r_{0}\right) \varphi_{20}^{2} \sqrt{\frac{L-r_{0}}{L-r_{1}(t)}} \cos \left(2 \sqrt{\frac{g}{L-r_{1}(t)}} t\right)\right) .
\end{aligned}
$$

Numerical estimation of the function (4.18) shows that it is really very small in comparison to $r_{1}(t)$ and it may play an essential role only in the case when the function $r_{1}(t)$ is close to its equilibrium value.

\section{Conclusion}

In the present paper we have analyzed an influence of oscillations on the Atwood machine motion in the case when both bodies are permitted to oscillate in a plane and the system has three degrees of freedom. To avoid an influence of a mass and size of the pulley and changing the length of the thread between the body $m_{1}$ and pulley owing to winding the thread on the pulley we have considered the generalized Atwood machine with two small massless pulleys shown in Fig. 1. Note that in this case the system motion does not change if the bodies oscillate in different vertical planes.

Doing necessary calculation, we have shown that even small oscillations of the bodies can completely modify a motion of the system, while the simple Atwood machine demonstrates only the uniformly accelerated motion of the bodies. We have shown that an averaged translational motion of the system may be described by simplified differential Eq. (4.14) which helps to understand qualitatively its behaviour. Comparing the obtained results with the numerical solutions of the exact equations of motion have demonstrated validity of the proposed approach.

It should be noted that the Atwood machine is a classical example of a physical system which seems to be quite simple but only in the case when all assumptions simplifying its motion are fulfilled. In practice it is quite difficult to avoid oscillations of the bodies and this stimulates investigation of the corresponding mathematical models which in fact turn out to be rather complicated. Fortunately, application of the modern computer algebra systems such as Wolfram Mathematica, for example, helps a lot in analyzing such problems and promotes development of physical intuition and better understanding of the subject.

Acknowledgements Many thanks to prof. Haiduke Sarafian for interesting discussion of the problem and useful remarks.

Open Access This article is distributed under the terms of the Creative Commons Attribution 4.0 International License (http:// creativecommons.org/licenses/by/4.0/), which permits unrestricted use, distribution, and reproduction in any medium, provided you give appropriate credit to the original author(s) and the source, provide a link to the Creative Commons license, and indicate if changes were made.

\section{References}

1. Atwood, G.: A Treatisa on the Rectilinear Motion and Rotation of Bodies. Cambridge University Press, Cambridge (1784)

2. Tufillaro, N.B., Abbott, T.A., Griffiths, D.J.: Swinging Atwood's machine. Amer. J. Phys. 52, 895-903 (1984)

3. Tufillaro, N.B.: Motions of a swinging Atwood's machine. J. Physique 46, 1495-1500 (1985)

4. Tufillaro, N.B.: Integrable motion of a swinging Atwood's machine. Am. J. Phys. 54, 142-143 (1986)

5. Casasayas, J., Nunes, T.A., Tufillaro, N.B.: Swinging Atwood's machine: integrability and dynamics. J. Physique 51, 1693-1702 (1990)

6. Yehia, H.M.: On the integrability of the motion of a heavy particle on a tilted cone and the swinging Atwood's machine. Mech. R. Commun. 33(5), 711-716 (2006)

7. Pujol, O., Pérez, J.P., Ramis, J.P., Simo, C., Simon, S., Weil, J.A.: Swinging Atwood machine: experimental and numerical results, and a theoretical study. Physica D 239(12), 1067-1081 (2010)

8. Zeleny, E.: Swinging Atwood's machine (2013). http://demonstrations.wolfram.com/SwingingAtwoodsMachine/. Accessed 13 Mar 2013 (2013)

9. Sarafian, H., Nenette, H.: Characteristics of a two-body holonomic constraint mechanical system. World J. Mech. 7, 161-166 (2017) 
10. Prokopenya, A.N.: Motion of a swinging Atwood's machine: simulation and analysis with Mathematica. Math. Comput. Sci. 11, 417-425 (2017)

11. Wolfram, S.: An elementary introduction to the Wolfram Language, 2nd editionn, 2nd edn. Wolfram Media, Champaign (2017)

12. Goldstein, H., Poole, C., Safko, J.: Classical Mechanics, 3rd edn. Addison Wesley, Reading, MA (2000) 